\title{
On farthest Voronoi cells
}

\author{
M. A. Goberna ${ }^{1}$ \\ Dep. of Mathematics, Universidad de Alicante, San Vicente del Raspeig, 03080, \\ Spain; email:mgoberna@ua.es \\ J. E. Martnez-Legaz \\ Dep. of Economics and Economic History, Universitat Autnoma de Barcelona, \\ and BGSMath, Spain; email: JuanEnrique.Martinez.Legaz@uab.cat
}

\author{
M. I. Todorov
}

Dep. of Physics and Mathematics, UDLAP, Puebla, MX. On leave from IMI-BAS, Sofia, Bulgaria;. email: maxim.todorov@udlap.mx

\begin{abstract}
Given an arbitrary set $T$ in the Euclidean space $\mathbb{R}^{n}$, whose elements are called sites, and a particular site $s$, the farthest Voronoi cell of $s$, denoted by $F_{T}(s)$, consists of all points which are farther from $s$ than from any other site. In this paper we study farthest Voronoi cells and diagrams corresponding to arbitrary (possibly infinite) sets. More in particular, we characterize, for a given arbitrary set $T$, those $s \in T$ such that $F_{T}(s)$ is nonempty and study the geometrical properties of $F_{T}(s)$ in that case. We also characterize those sets $T$ whose farthest Voronoi diagrams are tesselations of the Euclidean space, and those sets that can be written as $F_{T}(s)$ for some $T \subset \mathbb{R}^{n}$ and some $s \in T$.
\end{abstract}

Key words: Farthest Voronoi cells. Linear inequality systems. Boundedly exposed points.

Mathematics Subject Classification 2010: 15A39, 51M20, 52C22.

\section{Introduction}

Let $T \subset \mathbb{R}^{n}$, with $n \geq 1$, be a set whose elements are called Voronoi sites. The Voronoi cell of $s \in T$, denoted by $V_{T}(s)$, consists of all points closer to

$\overline{1}$ Corresponding author. 
$s$ than to any other site, i.e.,

$$
V_{T}(s):=\left\{x \in \mathbb{R}^{n}: d(x, s) \leq d(x, t), \forall t \in T\right\}
$$

where $d$ denotes the Euclidean distance on $\mathbb{R}^{n}$. Analogously, the farthest Voronoi cell of $s$, denoted by $F_{T}(s)$, is formed by those points which are farther from $s$ than from any other site, i.e.,

$$
F_{T}(s):=\left\{x \in \mathbb{R}^{n}: d(x, s) \geq d(x, t), \forall t \in T\right\}
$$

The families of sets $\left\{V_{T}(s), s \in T\right\}$ and $\left\{F_{T}(s), s \in T\right\}$ are called Voronoi diagram and farthest Voronoi diagram of $T$, respectively. If $T$ is a singleton, both diagrams trivially coincide with $\left\{\mathbb{R}^{n}\right\}$. So, we can assume that $T$ is not a singleton.

According to Michael Shamos' memories [21], the nearest Voronoi diagrams were rediscovered in 1974 by his collaborator Dan Hoey, a Yale undergraduate in Computer Science, who called them proximal polygons. Shamos found that they had been already discovered by Voronoi in 1908, and rediscovered independently many times since, so they decided to change their name to the above one. They also introduced the concept of farthest Voronoi diagram, obtaining algorithms for the construction of both types of diagrams that appeared in 1978, at Shamos Ph.D. Thesis [20], which was completed the next year with the proof of Shamos' conjecture that there is no real difference, from the computational point of view, between near and far. Shamos Ph.D. Thesis provided the starting stuff for the first published textbook on computational geometry [18].

There exists a vast literature on Voronoi cells and diagrams of finite sets due to their many applications in computational geometry, operations research, data compression, economics, marketing, etc. Farthest Voronoi cells and diagrams are not so popular, but they also have applications to computational geometry (see, e.g., [8] and [18]) and operations research, where they have been used to solve the so-called central path problem [27], to compute the min-power centre of a finite set of nodes in the Euclidean plane when cost is a quadratic function [4], or to solve the weighted one-center location problem (consisting in finding the minimum covering ball of a finite set of points in $\mathbb{R}^{n}$, with a positive weight corresponding to each point) [9].

Both types of cells and diagrams have been extended in several directions, for instance, replacing the Euclidean distance with another distance or pseudo distance (as the so-called travel time distance in [3]), and replacing in the definition of cells the given point site $s$ with segments (as in [1] and [2]) or with polygons (as in [6] and [7]), in which case the cells are no longer connected sets. Another extension can be obtained by relaxing the finiteness assumption 
on the set of sites $T$. This was already done by Delauney in the 1930s, who considered Voronoi cells and diagrams of infinite discrete sets (i.e., sets without accumulation points) in a work oriented towards crystallography [10]. More recently, [25] studied Voronoi cells of discrete sets by exploiting the linear representation of $V_{T}(s)$, while [14] and [15] extended this study to Voronoi cells of arbitrary infinite sets.

To the best of our knowledge, no previous work has considered farthest Voronoi cells and diagrams of infinite sets; this is the objective of the present paper, where we also use the linear algebra approach through the linear representation of $F_{T}(s)$. Our study reveals that the main difference between Voronoi cells and farthest Voronoi cells has to do with their nonemptiness and boundedness. In fact, $V_{T}(s)$ is always nonempty and may be bounded or not, while $F_{T}(s)$ is almost always empty (e.g., whenever $T$ is unbounded or when it is bounded but $s$ is not an extreme point of $\operatorname{conv} T$ ) and, when it is nonempty, it is necessarily unbounded. This is the reason why the paper is mostly focused on the conditions guaranteeing that $F_{T}(s) \neq \emptyset$. Since any discrete infinite set is unbounded, in contrast with [15] (on Voronoi cells of infinite sets), we do not consider in this paper farthest Voronoi cells of discrete sets.

The paper is organized as follows. Section 2 introduces the notation to be used along the paper and provides immediate consequences of three different representations of $F_{T}(s)$. Section 3 analyzes the relationships between $F_{T}(s)$ and the normal cone to the convex hull of $T$ at $s$, from which we obtain useful geometric information on the farthest Voronoi cells. Section 4 characterizes, for a given set $T$, those sites $s$ whose corresponding farthest Voronoi cells $F_{T}(s)$ are nonempty and those sets $T$ whose farthest Voronoi diagram is a tesselation of $\mathbb{R}^{n}$. Finally, Section 5 characterizes those subsets of $\mathbb{R}^{n}$ that are the farthest Voronoi cell $F_{T}(s)$ for some compact convex set $T$ and some $s \in T$, providing the farthest counterpart for the inverse problem solved in [14] for Voronoi cells.

\section{Basic results}

We first introduce the notation to be used along the paper. The sets of nonnegative and nonpositive real numbers are denoted by $\mathbb{R}_{+}$and $\mathbb{R}_{-}$, respectively. The zero vector in $\mathbb{R}^{n}$ is denoted by $0_{n}$ and the closed ball centered at $x \in \mathbb{R}^{n}$ and radius $r \in[0,+\infty]$ by $B(x, r)$ (notice that we allow $r$ to be 0 , in which case the "closed ball" $B(x, r)$ obviously degenerates to the singleton of its center, or $+\infty$, in which case $\left.B(x, r)=\mathbb{R}^{n}\right)$. Given $X \subset \mathbb{R}^{n}$, we denote by conv $X$, cone $X$, aff $X$, int $X$, and $\mathrm{cl} X$ the convex hull of $X$, the convex conical hull of $X$, the affine hull of $X$, the interior of $X$, and the closure of $X$, respectively. Given a nonempty convex set $X \subset \mathbb{R}^{n}$, its dimension and its 
recession cone are denoted by $\operatorname{dim} X$ and $0^{+} X$, respectively. The normal cone to a convex set $X \subset \mathbb{R}^{n}$ at $\bar{x} \in X$ is

$$
N_{X}(\bar{x}):=\left\{y \in \mathbb{R}^{n}:\langle x-\bar{x}, y\rangle \leq 0, \forall x \in X\right\}
$$

The support function of a nonempty set $X \subset \mathbb{R}^{n}$ is $\delta_{X}^{*}: \mathbb{R}^{n} \rightarrow \mathbb{R} \cup\{+\infty\}$, defined by $\delta_{X}^{*}(x):=\sup _{y \in X}\langle y, x\rangle$. It is known that $\delta_{X}^{*}$ is a lower semicontinuous positively homogeneous convex function. Moreover, $\delta_{X}^{*}$ is finite-valued (and so continuous on $\mathbb{R}^{n}$ ) whenever $X$ is bounded.

We now express the farthest Voronoi cells in tree different ways from which we obtain some immediate consequences. For each point $s$ of a given set $T \subset \mathbb{R}^{n}$, called the set of sites, we can express the farthest Voronoi cell as

$$
F_{T}(s)=\left\{x \in \mathbb{R}^{n}:\|x-s\| \geq\|x-t\|, \forall t \in T\right\} .
$$

Equivalently, one has

$$
F_{T}(s)=\left\{x \in \mathbb{R}^{n}: T \subset B(x,\|s-x\|)\right\}
$$

and, observing that the inequality in (1) can be replaced by $\|x-s\|^{2} \geq$ $\|x-t\|^{2}$, simple algebraic calculus yields

$$
F_{T}(s)=\left\{x \in \mathbb{R}^{n}: 2\langle t-s, x\rangle \geq\|t\|^{2}-\|s\|^{2}, \forall t \in T\right\} .
$$

Our first results are direct consequences of (1).

Proposition 1 If $T, T^{\prime} \subset \mathbb{R}^{n}$ and $s \in T \cap T^{\prime}$, one has $F_{T}(s) \cap F_{T^{\prime}}(s)=$ $F_{T \cup T^{\prime}}(s)$.

Corollary 2 If $T \subset T^{\prime} \subset \mathbb{R}^{n}$ and $s \in T$, then one has $F_{T^{\prime}}(s) \subset F_{T}(s)$.

Proposition 3 For $T \subset \mathbb{R}^{n}$ and $s \in T$, one has $F_{T}(s)=s+F_{T-s}\left(0_{n}\right)$.

Proof. Denoting $y:=x-s$ and $u:=t-s$,

$$
F_{T}(s)=\left\{y+s \in \mathbb{R}^{n}:\|y\| \geq\|y-u\|, \forall u+s \in T\right\}=s+F_{T-s}\left(0_{n}\right) .
$$

From (2), one easily obtains the following results.

Proposition 4 For $T \subset \mathbb{R}^{n}$ and $s \in T$, one has:

(i) If $F_{T}(s) \neq \emptyset$ then $T$ is bounded.

(ii) $F_{T}(s)=F_{\operatorname{clconv} T}(s)$. 
Proof. (i) Picking an arbitrary $x \in F_{T}(s),(2)$ shows that $T$ is contained in a ball centered at $x$.

(ii) It follows from (2), as

$$
T \subset B(x,\|s-x\|) \Longleftrightarrow \operatorname{cl} \operatorname{conv} T \subset B(x,\|s-x\|) .
$$

Definition 5 A nonempty set $T \subset \mathbb{R}^{n}$ is said to have the covering property if $\bigcup_{s \in T} F_{T}(s)=\mathbb{R}^{n}$.

Proposition 6 For any nonempty set $T \subset \mathbb{R}^{n}$, one has:

(i) If $T$ is compact, then it has the covering property.

(ii) If $T$ has the covering property, then it is bounded.

Proof. (i) Let $x \in \mathbb{R}^{n}$. Since the maximum distance from $x$ to the points of $T$ is attained at some $s$, this is a farthest site to $x$ in $T$ and, so, $x \in F_{T}(s)$.

Statement (ii) follows from Proposition 4(i).

As a consequence of Proposition 11(ii) below, all farthest Voronoi cells of open balls are empty. Thus, the compactness assumption in Proposition 6 cannot be replaced by the weaker one that $T$ is bounded.

Proposition 7 If $T \subset T^{\prime} \subset \mathbb{R}^{n}$, with $T$ and $T^{\prime}$ being compact, then the following equivalence holds:

$$
F_{T}(s)=F_{T^{\prime}}(s) \forall s \in T \Leftrightarrow T^{\prime} \subset \operatorname{conv} T \text {. }
$$

Proof. To prove $[\Rightarrow]$, let $B(x, r)$ be a closed ball containing $T$. By the covering property, there exists $s \in T$ such that $x \in F_{T}(s)$. Clearly, $\|s-x\| \leq r$ and, by the equality $F_{T}(s)=F_{T^{\prime}}(s)$, we have $x \in F_{T^{\prime}}(s)$. We thus obtain $T^{\prime} \subset$ $B(x,\|s-x\|) \subset B(x, r)$, which shows that $T^{\prime}$ is contained in the intersection of all those closed balls that contain $T$. According to the Mazur intersection property ([16], [17]) that intersection coincides with conv $T$.

The converse implication is an immediate consequence of Proposition 4(ii) combined with Corollary 2.

We now consider the partial ordering $\preccurlyeq$ defined on the class of compact subsets of $\mathbb{R}^{n}$ as follows:

$$
T \preccurlyeq T^{\prime} \Leftrightarrow T \subset T^{\prime} \text { and } F_{T}(s)=F_{T^{\prime}}(s) \forall s \in T \text {. }
$$


Corollary 8 If $T \subset \mathbb{R}^{n}$ is compact, then conv $T$ is the largest compact set $T^{\prime}$, for $\preccurlyeq$, such that $T \preccurlyeq T^{\prime}$.

Proof. It immediately follows from Propositions 4(ii) and 7.

Corollary 9 A compact set $T \subset \mathbb{R}^{n}$ is a maximal element for $\preccurlyeq$ if and only if it is convex.

Proof. It is an easy consequence of Corollary 8.

The next consequence of (3) implies that one can replace the covering property of $T$ in Proposition 6 by the stronger condition that the farthest Voronoi diagram of $T$ is a tesselation of $\mathbb{R}^{n}$, that is, the union of the family $\left\{F_{T}(s), s \in T\right\}$ is the whole of $\mathbb{R}^{n}$ and its corresponding family of topological interiors, $\left\{\operatorname{int} F_{T}(s), s \in T\right\}$, is formed by pairwise disjoint sets.

Proposition 10 For $T \subset \mathbb{R}^{n}$ and $s_{1}, s_{2} \in T$ such that $s_{1} \neq s_{2}$, one has $\operatorname{int} F_{T}\left(s_{1}\right) \cap \operatorname{int} F_{T}\left(s_{2}\right)=\emptyset$.

Proof. Let $x \in F_{T}\left(s_{1}\right) \cap F_{T}\left(s_{2}\right)$. By (3), one has $2\left\langle s_{2}-s_{1}, x\right\rangle \geq\left\|s_{2}\right\|^{2}-\left\|s_{1}\right\|^{2}$ and $2\left\langle s_{1}-s_{2}, x\right\rangle \geq\left\|s_{1}\right\|^{2}-\left\|s_{2}\right\|^{2}$, so that $x$ belongs to the hyperplane $H:=$ $\left\{x \in \mathbb{R}^{n}: 2\left\langle s_{2}-s_{1}, x\right\rangle=\left\|s_{2}\right\|^{2}-\left\|s_{1}\right\|^{2}\right\}$. Thus

$$
\operatorname{int} F_{T}\left(s_{1}\right) \cap \operatorname{int} F_{T}\left(s_{2}\right) \subset F_{T}\left(s_{1}\right) \cap F_{T}\left(s_{2}\right) \subset H \text {, }
$$

which implies int $F_{T}\left(s_{1}\right) \cap \operatorname{int} F_{T}\left(s_{2}\right)=\emptyset$.

We also get a geometric interpretation of $F_{T}(s)$ from its linear representation (3). Given $t \in T \backslash\{s\}$, the hyperplane of equation $2\langle t-s, x\rangle=\|t\|^{2}-\|s\|^{2}$ is orthogonal to the segment $[s, t]$ at its mid point $\frac{t+s}{2}$, and determines two halfspaces, one containing $s$,

$$
H_{t}^{\epsilon}(s):=\left\{x \in \mathbb{R}^{n}: 2\langle t-s, x\rangle \leq\|t\|^{2}-\|s\|^{2}\right\},
$$

and another one which does not contain it,

$$
H_{t}^{\notin}(s):=\left\{x \in \mathbb{R}^{n}: 2\langle t-s, x\rangle \geq\|t\|^{2}-\|s\|^{2}\right\} .
$$

Then,

$$
F_{T}(s)=\bigcap_{t \in T \backslash\{s\}} H_{t}^{\notin}(s),
$$

while the Voronoi cell of $s$ is

$$
V_{T}(s):=\left\{x \in \mathbb{R}^{n}:\|x-s\| \leq\|x-t\|, \forall t \in T\right\}=\bigcap_{t \in T \backslash\{s\}} H_{t}^{\in}(s) \ni s .
$$

Obviously, $V_{T}(s) \neq \emptyset$ for all $s \in T$ while $F_{T}(s)$ may be empty. 
Proposition 11 For $T \subset \mathbb{R}^{n}$ and $s \in T$, one has $F_{T}(s) \neq \emptyset$ if and only if

$$
\left(\begin{array}{c}
0_{n} \\
1
\end{array}\right) \notin \operatorname{clcone}\left\{\left(\begin{array}{c}
2(t-s) \\
\|t\|^{2}-\|s\|^{2}
\end{array}\right), t \in T\right\} .
$$

Proof. It follows from the generalized Gale's Theorem [13, Corollary 3.1.1] applied to the linear system in (3).

Example 12 Let $T=[-1,1]^{2}$ and let $T^{\prime}:=\{-1,1\}^{2}$ (the set of extreme points of $T)$. According to Proposition 4(ii), $F_{T}(s)=F_{T^{\prime}}(s)$ for all $s \in T^{\prime}$. Then, by (4), applied to $T^{\prime}$,

$$
\begin{aligned}
F_{T}(1,1) & =H_{(-1,1)}^{\notin}(1,1) \cap H_{(1,-1)}^{\notin}(1,1) \cap H_{(-1,-1)}^{\notin}(1,1) \\
& =\left\{x \in \mathbb{R}^{2}: x_{1} \leq 0, x_{2} \leq 0, x_{1}+x_{2} \leq 0\right\}=\mathbb{R}_{-}^{2} .
\end{aligned}
$$

Analogously, $F_{T}(-1,1)=\mathbb{R}_{+} \times \mathbb{R}_{-}, F_{T}(-1,-1)=\mathbb{R}_{+}^{2}$, and $F_{T}(1,-1)=\mathbb{R}_{-} \times$ $\mathbb{R}_{+}$, while $F_{T}(s)=\emptyset$ for all $s \in T \backslash T^{\prime}$. So, the farthest Voronoi diagram of $T$ is formed by the four quadrants of the plane (a tesselation of $\mathbb{R}^{2}$ ) together with an infinite number of copies of the empty set.

Example 13 Let $T=B\left(0_{2}, 1\right)$. One can easily see that $F_{T}(s)=\emptyset$ for all $s$ such that $\|s\|<1$. Let $s \in T$ be such that $\|s\|=1$. Then, $\left\{H_{t}^{\notin}(s): t \in T \backslash\{s\}\right\}$ is the family of all halfplanes not containing $s$ whose boundary contains $0_{n}$. So, $F_{T}(s)=\{-\lambda s: \lambda \geq 0\}$ (a ray, that is, a closed halfine emanating from $0_{2}$ ) and the farthest Voronoi diagram constitutes a tesselation of $\mathbb{R}^{2}$.

\section{Farthest Voronoi cells and normal cones}

We will use the following result (also valid for locally convex spaces) to get a fourth characterization of the elements of $F_{T}(s)$.

Lemma 14 (cf. [22, Theorem 3.1]) Let $f$ be a continuous convex function on $\mathbb{R}^{n}$ and $T$ be a bounded subset of $\mathbb{R}^{n}$ satisfying

$$
\inf f\left(\mathbb{R}^{n}\right)<\sup f(T) \text {. }
$$

For an element $t_{0} \in T$ one has $f\left(t_{0}\right)=\sup f(T)$ if and only if there exists a linear function $\Phi_{0} \neq 0$ such that

$$
\Phi_{0}\left(t_{0}\right)=\sup \left\{\Phi_{0}(z): z \in \mathbb{R}^{n}, f(z) \leq \sup f(T)\right\} .
$$


Proposition 15 For $T \subset \mathbb{R}^{n}, s \in T$ and $x \in \mathbb{R}^{n}$, the following equivalence holds:

$$
x \in F_{T}(s) \Leftrightarrow \exists y \in \mathbb{R}^{n} \text { such that }\|y\|=1 \text { and }\langle s, y\rangle=\delta_{B\left(x, \sup _{t \in T}\|t-x\|\right)}^{*}(y) \text {. }
$$

Proof. If $T$ is unbounded, then equivalence (5) holds vacuously true in view of Proposition 4(i) and the equality $\sup _{t \in T}\|t-x\|=+\infty$. We will assume that $T$ is not a singleton, since otherwise it is obvious that equivalence (5) holds true. Let $x \in \mathbb{R}^{n}$ and $f:=\|\cdot-x\|$. If $x \in F_{T}(s)$, recalling (1),

$$
\max f(T)=f(s)=\|x-s\|>0=\inf f\left(\mathbb{R}^{n}\right) .
$$

Then, by Lemma 14 , with $t_{0}=s$, and (2), there exists $y \in \mathbb{R}^{n}$ such that $\|y\|=1$ and

$$
\langle y, s\rangle=\sup \left\{\langle y, z\rangle: z \in \mathbb{R}^{n},\|z-x\| \leq \max f(T)\right\}=\delta_{B(x, \max f(T))}^{*}(y) .
$$

The argument is reversible.

The following results describe the relationship between $F_{T}(s)$ and $N_{\operatorname{conv} T}(s)$.

Proposition 16 For $T \subset \mathbb{R}^{n}$ and $s \in T$, one has

$$
F_{T}(s) \subset s-N_{\operatorname{conv} T}(s)
$$

Proof. If $T$ is unbounded, then $F_{T}(s)=\emptyset$ by Proposition 4(i). We can assume that $F_{T}(s) \neq \emptyset$. Let $x \in F_{T}(s)$, and take $y$ as in (5). We have

$$
\begin{aligned}
\|s-x\| & =\max _{t \in T}\|t-x\|=\left\langle x+\left(\max _{t \in T}\|t-x\|\right) y, y\right\rangle-\langle x, y\rangle \\
& \leq \delta_{B\left(x, \max _{t \in T}\|t-x\|\right)}^{*}(y)-\langle x, y\rangle=\langle s-x, y\rangle \leq\|s-x\|,
\end{aligned}
$$

hence

$$
\langle s-x, y\rangle=\delta_{B\left(x, \max _{t \in T}\|t-x\|\right)}^{*}(y)-\langle x, y\rangle=\|s-x\|,
$$

which implies that $s-x=\|s-x\| y$. Therefore, for $t \in \operatorname{conv} T$, in view of (2) and (5) we have

$$
\begin{aligned}
\langle t-s, x-s\rangle & =-\|x-s\|\langle t-s, y\rangle \\
& \geq-\|x-s\|\left(\delta_{B\left(x, \max _{t \in T}\|t-x\|\right)}^{*}(y)-\langle s, y\rangle\right)=0 .
\end{aligned}
$$

This implies that $x-s \in-N_{\operatorname{conv} T}(s)$, whereby $x \in s-N_{\operatorname{conv} T}(s)$.

The inclusions (6) are also strict in Example 13. 
Proposition 17 If $T \subset \mathbb{R}^{n}$ and $s \in T$ are such that $F_{T}(s) \neq \emptyset$, then

$$
0^{+} F_{T}(s)=-N_{\operatorname{conv} T}(s)
$$

and

$$
\operatorname{dim} F_{T}(s)=\operatorname{dim} N_{\operatorname{conv} T}(s)
$$

Proof. The inclusion $[\subset]$ in (7) is an immediate consequence of (6). To prove the opposite inclusion, let $d \in N_{\operatorname{conv} T}(s)$ and $x \in F_{T}(s)$. For every $t \in T$, one has

$$
\begin{aligned}
\|x-d-t\|^{2} & =\|x-t\|^{2}+\|d\|^{2}-2\langle x-t, d\rangle \\
& \leq\|x-s\|^{2}+\|d\|^{2}-2\langle x-t, d\rangle=\|x-d-s\|^{2}+2\langle t-s, d\rangle \\
& \leq\|x-d-s\|^{2}
\end{aligned}
$$

which shows that $x-d \in F_{T}(s)$; hence $-d \in 0^{+} F_{T}(s)$, as we wanted to prove. In order to prove (8), pick $x \in F_{T}(s)$. By (7) and (6), we have

$$
\begin{aligned}
x-N_{\operatorname{conv} T}(s) & \subset F_{T}(s)-N_{\operatorname{conv} T}(s)=F_{T}(s)+0^{+} F_{T}(s)=F_{T}(s) \\
& \subset s-N_{\operatorname{conv} T}(s),
\end{aligned}
$$

from which the equation (8) readily follows.

The next concept helps to understand the nature of the farthest Voronoi cells.

Definition 18 (cf. [26]) Let $X \subset \mathbb{R}^{n}$ and $x \in \mathbb{R}^{n}$. One says that $X$ is coradiant with respect to $x$ if its complement is starshaped with respect to $x$; in other words, if whenever $y \in X$ and $\lambda \geq 1$ then $(1-\lambda) x+\lambda y \in X$.

It is very easy to see that, for a closed convex set $X$ and $x \in \mathbb{R}^{n} \backslash X$, the following equivalence holds true $[26$, Proposition $2.5,(\mathrm{a}) \Leftrightarrow(\mathrm{b})]$ :

$$
X \text { is coradiant with respect to } x \Leftrightarrow X-x \subset 0^{+} X \text {. }
$$

When $x:=0_{n}$, one simply says that $X$ is coradiant. The following equivalence is immediate too:

$X$ is coradiant with respect to $x \Leftrightarrow X-x$ is coradiant.

Since $F_{T-s}\left(0_{n}\right) \subset-N_{\operatorname{conv} T}(s)=0^{+} F_{T-s}\left(0_{n}\right), F_{T-s}\left(0_{n}\right)$ and $-N_{\text {conv } T}(s)$ are coradiant.

From Propositions 16 and 17 we next obtain inner and outer approximations to $N_{\text {conv } T}(s)$ in terms of $F_{T}(s)$. The outer approximation involves the concept 
of outer kernel of a set $X \subset \mathbb{R}^{n}$ (cf. [26, Definition 3.2]), that is, the set

$$
\text { oker } X:=\left\{x \in \mathbb{R}^{n}: x+t(y-x) \notin X, \forall y \in \mathbb{R}^{n} \backslash X, \forall t \in(0,1]\right\}
$$

Observe that, defining the kernel of a set $Y \subset \mathbb{R}^{n}$ as

$$
\operatorname{ker} Y:=\left\{y \in \mathbb{R}^{n}: y+t(x-y) \in Y, \forall x \in Y, \forall t \in(0,1]\right\}
$$

oker $X$ turns out to be the kernel of its complement, i.e., oker $X=\operatorname{ker}\left(\mathbb{R}^{n} \backslash X\right)$.

From the definition of outer kernel,

$$
\text { oker } X=\left\{x \in \mathbb{R}^{n}: x+\lambda(z-x) \in X, \forall z \in X, \forall \lambda \geq 1\right\} \text {, }
$$

in other words, for every $x \in \mathbb{R}^{n} \backslash X$ one has

$$
x \in \text { oker } X \Leftrightarrow X \text { is coradiant with respect to } x \text {. }
$$

Theorem 19 (Farthest Voronoi cells and normal cones) For $T \subset \mathbb{R}^{n}$ and $s \in T$, one has

$$
s-F_{T}(s) \subset N_{\operatorname{conv} T}(s) \subset \operatorname{oker} F_{T}(s)-s .
$$

Proof. If $T$ is unbounded, by Proposition 4(i) the inclusions in (12) read $\emptyset \subset N_{\text {conv } T}(s) \subset \mathbb{R}^{n}$. If it is a singleton, then (12) reads $\mathbb{R}^{n} \subset \mathbb{R}^{n} \subset \mathbb{R}^{n}$. We can thus assume that $T$ is bounded and different from $\{s\}$. Since the empty set is trivially coradiant with respect to every point, we assume that $F_{T}(s) \neq \emptyset$. Let $x \in s+N_{\operatorname{conv} T}(s)$. Since $s$ is the closest point to $s$ in conv $T$ and $T$ is not a singleton, we have $x \notin F_{T}(s)$. On the other hand, by Propositions 4(i), 16 and 17 , we have

$$
\begin{aligned}
F_{T}(s)-x & \subset F_{T}(s)-s-N_{\operatorname{conv} T}(s) \subset-N_{\operatorname{conv} T}(s)-N_{\operatorname{conv} T}(s) \\
& =-N_{\operatorname{conv} T}(s)=0^{+} F_{T}(s) .
\end{aligned}
$$

Hence, according to the equivalence $(9)$, the set $F_{T}(s)$ is coradiant with respect to $x$, which, in view of $(11)$, means that $x \in$ oker $F_{T}(s)$. Thus,

$$
s+N_{\operatorname{conv} T}(s) \subset \operatorname{oker} F_{T}(s)
$$

Combining (6) and (13) one gets (12).

In Example 12, (12) becomes the following double strict inclusion:

$$
\left[1,+\infty\left[^ { 2 } \varsubsetneqq \mathbb { R } _ { + } ^ { 2 } \varsubsetneqq \left[-1,+\infty\left[^{2} .\right.\right.\right.\right.
$$




\section{Nonemptiness of the farthest Voronoi cells}

Definition 20 Let $T \subset \mathbb{R}^{n}$ be convex. A point $s \in T$ is said to be a boundedly exposed point of $T$ if there exists $\varepsilon>0$ such that $F_{T \cap B(s, \varepsilon)}(s) \neq \emptyset$.

In Definition 20 we borrow the term "boundedly exposed" from Edelstein [11] because, as we will see below, in the case when $T$ is bounded our notion coincides with the one introduced by this author with the same name in the context of metric spaces.

Proposition 21 Every boundedly exposed point of a convex set $T \subset \mathbb{R}^{n}$ is an exposed point of $T$.

Proof. Since the result is obvious when $T$ is a singleton, we will assume that it is not. Let $s$ be a boundedly exposed point of $T$ and $\varepsilon>0$ be such that $F_{T \cap B(s, \varepsilon)}(s) \neq \emptyset$. Take $y \in F_{T \cap B(s, \varepsilon)}(s)$. Since $T$ is convex but not a singleton, $T \cap B(s, \varepsilon)$ is not a singleton either, which clearly implies that $y \neq s$. Consider the halfspace $H^{+}:=\left\{x \in \mathbb{R}^{n}:\langle s-y, x-s\rangle \geq 0\right\}$. We will prove that $T \cap H^{+}=\{s\}$. The inclusion [ $\supset$ ] is obvious. To prove the opposite inclusion, let $x \in T \cap H^{+}$and take $\left.\lambda \in\right] 0$, 1] such that $(1-\lambda) s+\lambda x \in B(s, \varepsilon)$; since $(1-\lambda) s+\lambda x \in T \cap B(s, \varepsilon)$ and $y \in F_{T \cap B(s, \varepsilon)}(s)$, we have

$$
\begin{aligned}
\|y-s\|^{2} & \geq\|y-((1-\lambda) s+\lambda x)\|^{2}=\|y-s+\lambda(s-x)\|^{2} \\
& =\|y-s\|^{2}+2 \lambda\langle y-s, s-x\rangle+\lambda^{2}\|s-x\|^{2},
\end{aligned}
$$

which, as $x \in H^{+}$, implies that $x=s$. We have thus proved the equality $T \cap H^{+}=\{s\}$, which shows that $s$ is an exposed point of $T$.

The relevance of boundedly exposed points regarding farthest Voronoi cells stems from the following theorem.

Theorem 22 (Nonemptiness of the farthest Voronoi cells) For $T \subset \mathbb{R}^{n}$ and $s \in T$, the following equivalence holds:

$F_{T}(s) \neq \emptyset \Longleftrightarrow T$ is bounded and $s$ is a boundedly exposed point of cl conv $T$.

Proof. The implication $[\Longrightarrow]$ follows from Proposition $4(\mathrm{i})$ and the equalities

$$
F_{(\operatorname{cl} \operatorname{conv} T) \cap B(x, r)}(s)=F_{\operatorname{cl} \operatorname{conv} T}(s)=F_{T}(s)
$$

(see Proposition 4(ii)), with $r$ denoting a positive number large enough for the inclusion $T \subset B(x, r)$ to hold.

Conversely, assume that $T$ is bounded and $s$ is a boundedly exposed point of 
$\operatorname{cl} \operatorname{conv} T$, and take $\varepsilon$ such that $F_{(\operatorname{clconv} T) \cap B(x, \varepsilon)}(s) \neq \emptyset$. Pick $x \in F_{(\operatorname{clconv} T) \cap B(s, \varepsilon)}(s)$. We claim that the family of open sets $\{\operatorname{int}(B(s+\lambda(x-s), \lambda\|s-x\|))\}_{\lambda>0}$ covers the set $(\operatorname{cl} \operatorname{conv} T) \backslash\{s\}$, and a fortiori the set $(\operatorname{cl} \operatorname{conv} T) \backslash \operatorname{int}(B(s, \varepsilon))$. Indeed, for $y \in(\operatorname{cl} \operatorname{conv} T) \backslash\{s\}$ and small enough $t>0$ we have, by (2),

$$
(1-t) s+t y \in(\operatorname{cl} \operatorname{conv} T) \cap B(s, \varepsilon) \subset B(x,\|s-x\|),
$$

hence $\langle y-s, x-s\rangle>0$. Then it is easy to check that

$$
y \in \operatorname{int} B(s+\lambda(x-s), \lambda\|s-x\|), \forall \lambda>\frac{\|y-s\|^{2}}{\langle y-s, x-s\rangle},
$$

which proves the claim. Therefore, since $(\operatorname{cl} \operatorname{conv} T) \backslash \operatorname{int} B(s, \varepsilon)$ is compact and the sets $\operatorname{int} B(s+\lambda(x-s), \lambda\|s-x\|)$ depend increasingly (in the sense of inclusion) on $\lambda$, for some $\lambda_{0}>0$ we have

$$
(\operatorname{clconv} T) \backslash \operatorname{int} B(s, \varepsilon) \subset \operatorname{int} B\left(s+\lambda_{0}(x-s), \lambda_{0}\|s-x\|\right),
$$

which, in view of (2), implies that

$$
s+\lambda_{0}(x-s) \in F_{\mathrm{cl} \operatorname{conv} T \backslash \operatorname{int} B(s, \varepsilon)}(s) .
$$

On the other hand, using Proposition 17 we obtain

$$
s-x \in N_{B(x,\|s-x\|)}(s) \subset N_{(\operatorname{cl} \operatorname{conv} T) \cap B(s, \varepsilon)}(s) \subset-0^{+} F_{(\operatorname{cl} \operatorname{conv} T) \cap B(s, \varepsilon)}(s) ;
$$

hence

$$
s+\lambda_{0}(x-s) \in F_{(\operatorname{cl} \operatorname{conv} T) \cap(B(s, \varepsilon))}(s) .
$$

Finally, by (14), (15) and Propositions 1 and 4(ii), we get

$$
\begin{aligned}
s+\lambda_{0}(x-s) & \in F_{(\operatorname{cl} \operatorname{conv} T) \backslash \operatorname{int} B(s, \varepsilon)}(s) \cap F_{(\operatorname{cl} \operatorname{conv} T) \cap(B(s, \varepsilon))}(s) \\
& =F_{\operatorname{cl} \operatorname{conv} T}(s)=F_{T}(s),
\end{aligned}
$$

which shows that $F_{T}(s) \neq \emptyset$.

As a first corollary of Theorem 22, we obtain that, for bounded sets, our notion of boundedly exposed point coincides with that of Edelstein [11].

Corollary 23 For any bounded set $T \subset \mathbb{R}^{n}$ and $s \in T$, the following equivalence holds:

$s$ is a boundedly exposed point of $\operatorname{cl} \operatorname{conv} T$

$\exists x \in \mathbb{R}^{n}$ such that $T \backslash\{s\} \subset \operatorname{int} B(x,\|s-x\|)$. 
Proof. The proof of [个] is immediate: from $T \backslash\{s\} \subset \operatorname{int} B(x,\|s-x\|)$ we easily deduce that $\operatorname{cl} \operatorname{conv} T \subset B(x,\|s-x\|)$, which, by (2) and Corollary 2, implies that $x \in F_{\text {cl conv } T}(s) \subset F_{(\operatorname{cl} \operatorname{conv} T) \cap B(s, 1)}(s)$, thus showing that $F_{(\mathrm{clconv} T) \cap B(s, 1)}(s) \neq \emptyset$ and hence that $s$ is a boundedly exposed point of cl conv $T$. Conversely, if $s$ is a boundedly exposed point of $\operatorname{cl} \operatorname{conv} T$ then, by Theorem 22, we have $F_{T}(s) \neq \emptyset$. Taking $y \in F_{T}(s)$, from (2) we deduce that $T \subset B(y,\|s-y\|)$, which implies that $T \backslash\{s\} \subset B(y,\|s-y\|) \backslash\{s\} \subset$ int $B(x,\|s-x\|)$ for $x:=2 y-s$.

The next corollary is the farthest Voronoi counterpart of the known fact that the Voronoi diagram of $T$ is a tesselation of $\mathbb{R}^{n}$ if and only if $T$ is closed [15, Proposition 1].

Corollary 24 The farthest Voronoi diagram of $T$ is a tesselation of $\mathbb{R}^{n}$ if and only if $T$ is bounded and contains all boundedly exposed points of cl conv $T$.

Proof. Assume that the farthest Voronoi diagram of $T$ is a tesselation of $\mathbb{R}^{n}$. Then, $T$ is bounded by Proposition 6(ii). We now prove that $T$ contains all boundedly exposed points of clconv $T$ by contradiction. Let $s$ be a boundedly exposed point of cl conv $T$ such that $s \notin T$. By Corollary 23, applied to $\operatorname{cl} \operatorname{conv} T$, there exists $x \in \mathbb{R}^{n}$ such that $(\operatorname{clconv} T) \backslash\{s\} \subset \operatorname{int} B(x,\|x-s\|)$. Let $s^{\prime} \in T$ be such that $x \in F_{T}\left(s^{\prime}\right)$. Then $\left\|x-s^{\prime}\right\|<\|x-s\|$, as $s^{\prime} \in$ $(\operatorname{clconv} T) \backslash\{s\}$, while $\|x-s\| \leq\left\|x-s^{\prime}\right\|$ by (1), also applied to $\operatorname{clconv} T$, and Proposition 4(ii). This contradiction shows that $x \notin \bigcup_{s^{\prime} \in T} F_{T}\left(s^{\prime}\right)$ and, so,

$T$ does not enjoy the covering property.

Conversely, if $T$ is bounded, by Theorem 22, the nonempty elements of the family $\left\{F_{T}(s), s \in T\right\}$ are those farthest Voronoi cells $F_{T}(s)$ such that $s \in T$ is a boundedly exposed point of $\operatorname{clconv} T$, for which $F_{T}(s)=F_{\operatorname{clconv} T}(s)$, again by Proposition 4(ii). Hence, the nonempty elements of the farthest Voronoi diagrams of $T$ and cl conv $T$ are the same, with cl conv $T$ satisfying the covering property by Proposition 6(i). The proof is complete recalling that the interiors of different farthest Voronoi cells are disjoint (Proposition 10).

Corollary 25 Every compact convex set in $\mathbb{R}^{n}$ is the closed convex hull of its boundedly exposed points.

Proof. This is an immediate consequence of Theorem 22 combined with a theorem due to Edelstein [12], according to which every closed and bounded subset $T$ of a uniformly Banach space with the Mazur intersection property is the closed convex hull of its farthest points (i.e., points $s$ such that $F_{T}(s) \neq \emptyset$ ).

In Examples 12 and 13, where cl conv $T=T$, any extreme point $s$ satisfies the 
characterization of the boundedly exposed points in Corollary 23 by taking $x=-s$. The following example shows that an exposed point need not be boundedly exposed.

Example 26 Consider the compact convex set

$$
T:=\left\{(x, y) \in \mathbb{R}^{2}: x^{4} \leq y \leq 1\right\}
$$

Clearly, the origin $(0,0)$ is an exposed point of $T$. To prove that it is not boundedly exposed, we will prove that $F_{T}(0,0)=\emptyset$; the conclusion will then follow from Theorem 22. Assume, to the contrary, that $F_{T}(0,0) \neq \emptyset$, and take $(x, y) \in F_{T}(0,0)$. By (2), one has $T \subset B\left((x, y), \sqrt{x^{2}+y^{2}}\right)$. Hence, for every $t \in[-1,1]$, from $\left(t, t^{4}\right) \in T$ we deduce that $(t-x)^{2}+\left(t^{4}-y\right)^{2} \leq x^{2}+y^{2}$, that $i s, t^{2}-2 t x+t^{8}-2 t^{4} y \leq 0$. Dividing this inequality by $|t|$, with $t \neq 0$, and setting successively $t \rightarrow 0^{+}$and $t \rightarrow 0^{-}$, one easily obtains that $x=0$, and hence $1+t^{6}-2 t^{2} y \leq 0$ for $t \in[-1,1] \backslash\{0\}$, which, setting $t \rightarrow 0$, yields an absurd conclusion. Therefore $F_{T}(0,0)=\emptyset$.

The celebrated Straszewicz's Theorem ([23], [19, Theorem 18.6]) asserts that the set of exposed points of a closed convex set $T \subset \mathbb{R}^{n}$ is dense in the set of extreme points of $T$. We next prove that this theorem remains true when exposed points are replaced by boundedly exposed points.

Theorem 27 (Density of the boundedly exposed points) For any closed convex set $T \subset \mathbb{R}^{n}$, the set of boundedly exposed points of $T$ is dense in the set of extreme points of $T$.

Proof. In view of Straszewicz's Theorem, it will be enough to prove that the set of boundedly exposed points of $T$ is dense in the set of exposed points of $T$. Let $x$ be an exposed point of $T$. We assume, without loss of generality, that $T$ is compact (we can replace, if necessary, $T$ with the intersection of a closed ball centered at $x$, since the exposed points of that intersection that are close enough to $x$ are necessarily exposed points of $T$, as one can easily see). Let $\varepsilon>0$. We have to prove the existence of a boundedly exposed point of $T$ in $\operatorname{int} B(x, \varepsilon)$. Take a supporting hyperplane $H$ of $T$ such that $T \cap H=\{x\}$, and let $p$ be the corresponding unit exterior normal vector to $T$ at $x$, i.e., $p \in N_{T}(x)$ and orthogonal to $H$. We now use an argument similar, though simpler, to one we have employed in the proof of Theorem 22. We claim that the family of open sets $\{\operatorname{int}(B(x-\lambda p, \lambda))\}_{\lambda>0}$ covers the set $T \backslash\{x\}$, and hence $T \backslash \operatorname{int} B(x, \varepsilon)$. Indeed, by the definition of $p$ we have $\langle p, y-x\rangle<0$ for all $y \in T \backslash\{x\}$. Then it is easy to check that $y \in \operatorname{int}(B(x-\lambda p, \lambda))$ for every $\lambda>\frac{\|y-x\|^{2}}{2\langle p, y-x\rangle}$, which proves the claim. Therefore, since $T \backslash \operatorname{int} B(x, \varepsilon)$ is compact and the sets int $(B(x-\lambda p, \lambda))$ depend increasingly (in the sense of 
inclusion) on $\lambda$, for some $\lambda_{0}>0$ we have

$$
T \backslash \operatorname{int} B(x, \varepsilon) \subset \operatorname{int}\left(B\left(x-\lambda_{0} p, \lambda_{0}\right)\right) .
$$

By Proposition 6(i), we have $x-\lambda_{0} p \in F_{T}(s)$ for some $s \in T$. Then $\left\|x-\lambda_{0} p-s\right\| \geq$ $\left\|x-\lambda_{0} p-x\right\|=\lambda_{0}$, hence $s \notin \operatorname{int} B\left(x-\lambda_{0} p, \lambda_{0}\right)$, which, by (16), implies that $s \in \operatorname{int} B(x, \varepsilon)$. To finish the proof, we just have to apply Theorem 22 , as it tells us that $s$ is a boundedly exposed point of $T$.

\section{Characterization of the farthest Voronoi cells}

We next aim at characterizing farthest Voronoi cells. In view of Proposition 3 , one just needs to consider the case when $s:=0_{n}$, in which case (3) reduces to

$$
F_{T}\left(0_{n}\right)=\left\{x \in \mathbb{R}^{n}:\langle t, x\rangle \geq \frac{\|t\|^{2}}{2}, \forall t \in T \backslash\left\{0_{n}\right\}\right\} .
$$

This representation shows that $F_{T}\left(0_{n}\right)$ is closed, convex and coradiant; moreover, $0_{n} \notin F_{T}\left(0_{n}\right)$ unless $T=\left\{0_{n}\right\}$. In order to prove that these properties characterize the farthest Voronoi cells of $0_{n}$ we need a known result that involves the concept of reverse polar of a set, which results of inverting the inequality $\langle x, y\rangle \leq 1$ in the definition of positive polar. Indeed, the reverse polar set $X^{0}$ of a nonempty set $X \subset \mathbb{R}^{n}$ is

$$
X^{0}:=\left\{y \in \mathbb{R}^{n}:\langle x, y\rangle \geq 1, \forall x \in X\right\} .
$$

Lemma 28 (cf. [26, Proposition 2.5, (a) $\Leftrightarrow(e)]$ ) A set $C \subset \mathbb{R}^{n}$ is convex, closed, coradiant and does not contain $0_{n}$ if and only if there exists a nonempty set $U \subset \mathbb{R}^{n} \backslash\left\{0_{n}\right\}$ such that

$$
C=U^{0}
$$

Proof. The equivalence obviously holds if $C=\emptyset$ (take, e.g., $U:=\{p,-p\}$, with $p \in \mathbb{R}^{n} \backslash\left\{0_{n}\right\}$ ), so we are going to assume that $C \neq \emptyset$. The "only if" statement being trivial, we will only prove its converse. Since $C$ is convex and closed, there exists $Q \subset\left(\mathbb{R}^{n} \backslash\left\{0_{n}\right\}\right) \times \mathbb{R}$ such that

$$
C=\left\{x \in \mathbb{R}^{n}:\langle a, x\rangle \geq b, \forall(a, b) \in Q\right\} .
$$

Since $C$ is coradiant, for every $x \in \mathbb{R}^{n}$ and $(a, b) \in Q$ we have $\langle a, x\rangle \geq 0$, and hence there is no loss of generality in assuming that $b \geq 0$. We can rewrite $C$ as follows:

$$
C=\left\{x \in \mathbb{R}^{n}:\langle a, x\rangle \geq 1 \forall a \in A_{+}, \text {and }\langle a, x\rangle \geq 0 \forall a \in A_{0}\right\} ;
$$

here $A_{+}:=\left\{\frac{1}{b} a:(a, b) \in Q, b>0\right\}$ and $A_{0}:=\left\{a \in \mathbb{R}^{n}:(a, 0) \in Q\right\}$. Since $0_{n} \notin C$, we have $A_{+} \neq \emptyset$. Pick $\bar{a} \in A_{+}$, and for each $a \in A_{0}$ replace the 
inequality $\langle a, x\rangle \geq 0$ with the following equivalent infinite collection of inequalities:

$$
\langle a+\varepsilon \bar{a}, x\rangle \geq \varepsilon, \forall \varepsilon>0 .
$$

Thus, (18) holds with $U:=A_{+} \cup\left\{\frac{1}{\varepsilon} a+\bar{a}: a \in A_{0}, \varepsilon>0\right\}$.

Theorem 29 (Characterization of the farthest Voronoi cells) For any nonempty set $C \varsubsetneqq \mathbb{R}^{n}$, one has $C=F_{T}(s)$ for some $T \subset \mathbb{R}^{n}$ containing $s$ if and only if it is convex, closed, coradiant with respect to $s$ and does not contain $s$.

Proof. In view of Proposition 3 and (10), we may assume, without loss of generality, that $s:=0_{n}$. Then, as we have already observed, the "only if" statement is an immediate consequence of (17). To prove the converse, use the representation (18) and define $T:=\left\{\frac{2}{\|u\|^{2}} u: u \in U\right\} \cup\left\{0_{n}\right\}$. Since $U \neq \emptyset$, the set $T$ is not a singleton. Using (17), it is easy to check that $F_{T}\left(0_{n}\right)=C$.

Example 30 Let $C:=\left\{(x, y) \in \mathbb{R}^{2}: x>0, x y \geq 1\right\}$. This set is convex, closed, coradiant and does not contain the origin. From the representation

$$
C=\left\{(x, y) \in \mathbb{R}^{2}: x+t^{2} y \geq 2 t, t>0\right\}
$$

one easily sees that (18) holds with $U:=\left\{\frac{1}{2}\left(\frac{1}{t}, t\right): t>0\right\}$. Hence, according to the proof of Theorem 29, for $T=\left\{\left(\frac{4 t}{1+t^{4}}, \frac{4 t^{3}}{1+t^{4}}\right): t>0\right\} \cup\left\{0_{2}\right\}$ one has $F_{T}\left(0_{2}\right)=C$.

Corollary 31 For any $s \in \mathbb{R}^{n}$, the mapping $T \mapsto F_{T}(s)$ is a bijection from the set of all compact convex subsets of $\mathbb{R}^{n}$ that contain $s$ as a boundedly exposed point onto the the set of all nonempty closed convex subsets of $\mathbb{R}^{n}$ that are coradiant with respect to $s$ and do not contain $s$.

Proof. It is an easy consequence of Theorems 22 and 29 together with Proposition 7 .

\section{Acknowledgments.}

The authors wish to thank an anonymous referee for her/his valuable comments and criticisms which helped us improve the manuscript. We are also grateful to Alberto Zaffaroni for helpful discussions on coradiant sets; in particular, for providing us with reference [26].

The first and second author were supported by Ministry of Science, Innovation and Universities of Spain and the European Regional Development Fund (ERDF) of the European Commission, Grants PGC2018-097960-B-C22 and PGC2018-097960-B-C21, respectively. The second author was also supported 
by the Severo Ochoa Programme for Centres of Excellence in R\&D [SEV2015-0563]. He is affiliated to MOVE (Markets, Organizations and Votes in Economics).

\section{References}

[1] S.W. Bae, Tight bound for farthest-color Voronoi diagrams of line segments, in: WALCOM: Algorithms and Computation, 40-51, Lecture Notes in Comput. Sci., 7157, Springer, Heidelberg, 2012.

[2] S.W. Bae, Tight bound and improved algorithm for farthest-color Voronoi diagrams of line segments, Comput. Geom. 47 (2014) 779-788.

[3] S.W. Bae, K.-Y. Chwa, Farthest Voronoi diagrams under travel time metrics (extended abstract). WALCOM: Algorithms and Computation, 28-39, Lecture Notes in Comput. Sci., 7157, Springer, Heidelberg, 2012.

[4] M. Brazil, C.J. Ras, D.A. Thomas, A geometric characterisation of the quadratic min-power centre, European J. Oper. Res. 233 (2014) 34-42.

[5] K.Q. Brown, Geometric Transformations for Fast Geometric Algorithms, Ph.D. Thesis, Department of Computer Science, Carnegie Mellon University, 1979.

[6] O. Cheong, H. Everett, M. Glisse, J. Gudmundsson, S. Hornus, S. Lazard, M. Lee, H.-S. Na, Farthest-polygon Voronoi diagrams, Comput. Geom. 44 (2011) 234-247.

[7] Y.K. Cheung, O. Daescu, M. Zivanic, Kinetic red-blue minimum separating circle, in: Combinatorial Optimization and Applications, 448-463, Lecture Notes in Comput. Sci., 6831, Springer, Heidelberg, 2011.

[8] M. de Berg, M. van Kreveld, M. Overmars, O. Schwarzkopf, Computational Geometry: Algorithms and Applications, Springer, New York-Secaucus, NJ, 1997.

[9] P.M. Dearing, P. Belotti, A.M. Smith, A primal algorithm for the weighted minimum covering ball problem in $\mathbb{R}^{n}$, TOP 24 (2016) 466-492.

[10] B. Delaunay, Sur la sphère vide: À la mémoire de Georges Voronoi (French), Izv. Akad. Nauk SSSR, Otdelenie Matematicheskih i Estestvennyh Nauk 7 (1934) 793-800.

[11] M. Edelstein, On some special types of exposed points of closed and bounded sets in Banach spaces. I, Indag. Math. 28 (1966) 360-363.

[12] M. Edelstein, Farthest points of sets in uniformly convex Banach spaces. Israel J. Math. 4 (1966) 171-176.

[13] M.A. Goberna, M.A. López, Linear Semi-Infinite Optimization, Wiley, Chichester, 1998. 
[14] M.A. Goberna, J.-E. Martínez-Legaz, V.N. Vera de Serio, The Voronoi inverse mapping, Linear Algebra Appl. 504 (2016) 248-271.

[15] M.A. Goberna, M.M.L. Rodríguez, V.N. Vera de Serio, Voronoi cells via linear inequality systems, Linear Algebra Appl. 436 (2012) 2169-2186.

[16] S. Mazur, Über schwache Konvergenz in den Räumen $\left(L^{p}\right)$ (German), Studia Math. 4 (1933) 128-133.

[17] R.R. Phelps, A representation theorem for bounded convex sets, Proc. Am. Math. Soc. 11 (1960) 976-983.

[18] F.P. Preparata, M.I. Shamos, Computational Geometry: An Introduction, Springer, New York, 1985.

[19] R.T. Rockafellar, Convex Analysis, Princeton University Press, Princeton, N.J., 1970.

[20] M. Shamos, Computational Geometry, Ph.D. Thesis, Department of Computer Science, Yale University, 1978. Available from University Microfilms, Ann Arbor, MI.

[21] M. Shamos, The Early Years of Computational Geometry - A Personal Memoir, Advances in Discrete and Computational Geometry (B. Chazelle, J. E. Goodman, and R. Pollack, eds.), Contemporary Mathematics, pp. 313-333, Amer. Math. Soc., Providence, 1998.

[22] I. Singer, Maximization of lower semi-continuous convex functionals on bounded subsets of locally convex spaces. I: Hyperplane theorems, Appl. Math. Optimiz. 5 (1979) 349-362.

[23] S. Straszewicz, Über exponierte Punkte abgeschlossener Punktmengen (German), Fundamenta Math. 24 (1935) 139-143.

[24] J. Tind, Blocking and antiblocking sets, Math. Program. 6 (1974) 157-166.

[25] I. Voigt, S. Weis, Polyhedral Voronoi cells, Beiträge Algebra Geom. 51 (2010) 587-598.

[26] A. Zaffaroni, Convex coradiant sets with a continuous concave cogauge, J. Convex Anal. 15 (2008) 325-343.

[27] Y. Zhu, J. Xu, On the central path problem, Theoret. Comput. Sci. 507 (2013) 83-99. 